DOI: http://doi.org/10.21698/simi.2018.ab12

\title{
PERMANGANATE ASSISTED DEGRADATION OF TEXTILE DYES IN ALKALINE MEDIA
}

Ioana Cristina Isac, Adina Raducan, Petruta Oancea

Department of Physical Chemistry, Faculty of Chemistry, University of Bucharest, 4-12 Elisabeta Boulevard, Bucharest, adina.raducan@g.unibuc.ro, Romania

Keywords: dye degradation, permanganate oxidation

\section{Introduction}

Synthetic and natural dyes are major components of the trade effluent from carpet manufacturing, paint, textile, tannery, paper and cosmetic industries, whose discharge in the environment often poses severe threat on the health and integrity of the ecosystem. Though they display significant structural diversity, the main chemical classes of dyes used at industrial scale are azo, indigoid, anthraquinone, sulfur, triphenylmethyl (trityl) and phthalocyanine. Most of them are highly toxic, displaying also mutagenic and carcinogenic long term effects; they are resistant to microbial attack and present high stability to light, heating, and oxidation. Even small amounts of dyes in water (up to $1 \mathrm{ppm}$ for some dyes), are enough to produce color which is totally undesirable for any kind of natural or surface water. There is a considerable demand for color-free effluents, but the color removal from wastewater may involve extremely difficult and costly treatments. The usual technologies for color removal include adsorption on inorganic/organic matrices, photocatalysis, oxidation and enzymatic decomposition. In this context, we investigated the oxidation with potassium permanganate in alkaline media as a pretreatment option for degradation of several textile dyes.

\section{Materials and methods}

$\mathrm{KMnO}_{4}$ (purity $\geq 99 \%$, ACS reagent) and $\mathrm{NaOH}$ (purity $\geq 97 \%$, ACS reagent) were purchased from Sigma and dissolved further in bi-distilled water. Volumetric solutions $(0.05$ and $0.5 \mathrm{M})$ of oxalic acid for $\mathrm{KMnO}_{4}$ titration were acquired from Sigma. Copper $\alpha$-phthalocyanine with $3 \%$ organically combined chlorine $(\mathrm{Cl}-\alpha \mathrm{CuPc}$, Heliogen Blue K6902) and copper $\beta$ - phthalocyanine ( $\beta \mathrm{CuPc}$, Heliogen Blue K7090) were purchased from BASF; textile dyes Basic violet 14, Direct Red 28, Acid Red 17, Acid Yellow 1, Acid blue 9 and Acid blue 64 were acquired from DOW Chemicals, Acid black 210, Acid brown 98, Acid red 119 from Sella.

Dye solution with excess of permanganate $(12 \mathrm{mM})$ and $\mathrm{NaOH}(50 \mathrm{mM})$, were allowed to react for at least $1 \mathrm{~h}$ at $56^{\circ} \mathrm{C}$. Under these conditions, the $\mathrm{pH}$ of the reaction mixtures ranged between 10 and 11.5 .

Ultraviolet-visible (UV-VIS) spectra of dye solutions before and during the degradation experiments were recorded between 200 and $900 \mathrm{~nm}$ using a Jasco V-530 spectrophotometer with a Peltier cell for temperature and stirring control. 


\section{INTERNATIONAL SYMPOSIUM "THE ENVIRONMENT AND THE INDUSTRY", SIMI 2018, BOOK OF ABSTRACTS}

\section{Results and conclusions}

The extent of dye degradation was estimated from the kinetic curve absorbance vs. time at the maximum absorption wavelength. The apparent first-order kinetic constants k' was estimated through non-linear regression by fitting the exponential decay equation $A=A_{0} \cdot \exp \left(-k^{\prime} t\right)$ on the experimental curves absorbance A $\lambda$ max vs. time. These values were used further to calculate the half-life of phthalocyanines in the degradation process as:

$t_{1 / 2}=\frac{\ln 2}{k^{\prime}}$

The degradation of textile dyes was also quantified by their degrees of decolourization (\%) calculated as:

$D(\%)=\frac{A_{0}-A_{\text {fin }}}{A_{0}} \cdot 100$

where $\mathrm{A}_{0}$ is the initial absorbance of the sample without permanganate and $\mathrm{A}_{\text {fin }}$ represents the final absorbance at the same wavelength after $4 \mathrm{~h}$ of freaction. The decolourization degrees are presented in Figure 1.

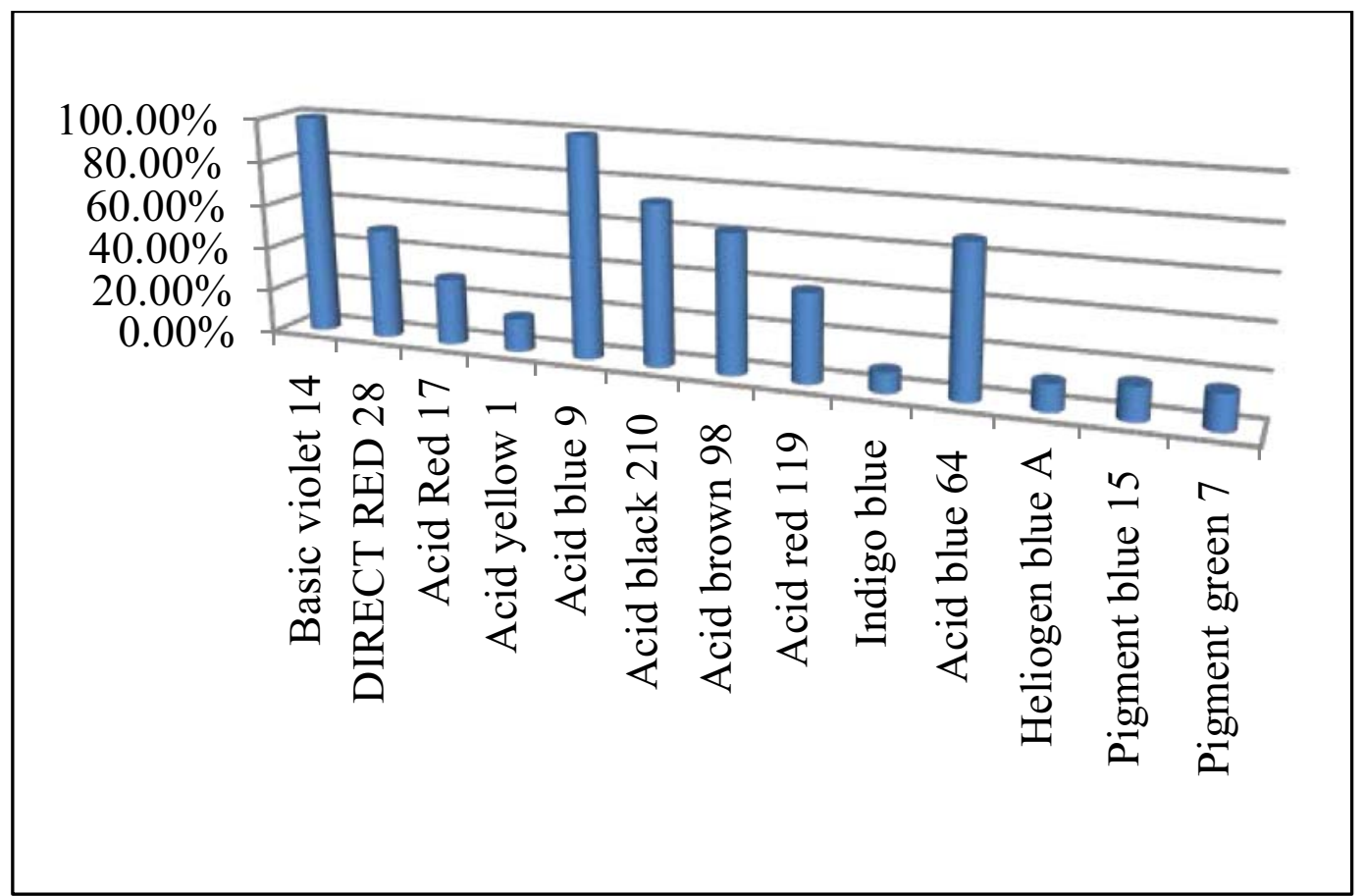

Figure 1. Decolourization degrees of selected dyes after $4 \mathrm{~h}$ reaction with permanganate in alkaline media

The time required for the complete fading ranged between 4 and $24 \mathrm{~h}$, depending on the initial concentration and type of dyes. 